\title{
QUOTIENTS IN NOETHERIAN LATTICE MODULES
}

\author{
J. A. JOHNSON ${ }^{1}$
}

AbSTRACT. In this paper we obtain a generalization of the fact that if $M$ is a maximal (proper) ideal of a Noetherian ring $R$, then the ring $M / M A$ is a vector space over $R / M$ for all ideals $A$ of the ring $R$.

1. Introduction. In a recent paper [1] Bogart proved the following result $([1$, Theorem 3.1$])$ :

(1.1) A lattice $L$ may be represented as a Noetherian lattice with the trivial multiplication if and only if $L$ is a finite-dimensional modular lattice in which every element except the unit element is a join of atoms.

Among other things, he wanted to use this result to prove a generalization of the well-known fact that if $M$ is a maximal (proper) ideal of a Noetherian ring $R$, then the ring $M / M A$ is a vector space over $R / M$ for all ideals $A$ of the ring $R$. His generalization stated that if $M$ is a maximal (proper) element of a Noetherian lattice, then the quotient sublattice $A / M A$ is a complemented modular lattice for all $A$ in $L$. The proof given is valid for local Noetherian lattices, and the hypothesis that $L$ is local, or at least that $A \leqq M$, is used in line 4 on p. 133 of Bogart's paper. Bogart did in fact intend for $L$ to be local in his theorem. In this paper we show that this hypothesis is needed in order to use Bogart's construction to prove the theorem, and show that an equally simple method does suffice to prove the theorem. We then develop enough of the theory of Noetherian lattice modules to use methods analogous to Bogart's to prove the following generalization of (1.1) and Bogart's Theorem 5.1.

(1.2) Let $L$ be a modular multiplicative lattice with maximal (proper) elements. Let $M$ be a complete modular lattice with more than one element. Then $M$ can be represented as a Noetherian $L$-module with the trivial multiplication if and only if $L$ has only one maximal element and each element of $M$ is a join of atoms.

(1.3) Let $L$ be a modular multiplicative lattice with maximal

Received by the editors May 5, 1970.

AMS 1969 subject classifications. Primary 0635, 0640; Secondary 1320.

Key words and phrases. Lattice, modular, multiplicative, complemented, lattice module, Noetherian.

1 This research was partially supported by the Jet Propulsion Laboratory, California Institute of Technology, under Contract No. NAS7-100, sponsored by the National Aeronautics and Space Administration. 
elements, let $M$ be a Noetherian $L$-module, let $m$ be a maximal element of $L$, and let $A$ be an arbitrary element of $M$. Then

(i) $A / m A$ is a Noetherian $L / m$-module,

(ii) $A / m A$ is a finite-dimensional complemented modular lattice.

2. Preliminary remarks. In this section we shall use the terminology of $\S 5$, Theorem 5.1 of [1]. Thus $L$ is a Noetherian lattice, $A$ is an arbitrary element of $L$ and $M$ is a maximal (proper) element of $L$. In the proof of Theorem 5.1 Bogart wants to show that the set $L^{\prime}$ consisting of $I$ and the elements of $A / A M$ forms a Noetherian lattice with the trivial multiplication. He defines the multiplication "o" in $L^{\prime}$ by $X \circ Y=X Y \bigvee A M$, for all $X, Y$ in $L^{\prime}$. If this is the trivial multiplication, then $X \circ Y=X Y \vee A M=A M$ for all $X, Y$ in $A / A M$. Thus $X Y \leqq A M$. In particular, $A A \leqq A M \leqq M$. Since $M$ is prime in $L$, it follows that $A \leqq M$. Thus this multiplication is the trivial multiplication if and only if $A \leqq M$.

Assume for a moment that there is a multiplication, denoted by "*", on $L^{\prime}$, such that $L^{\prime}$ is a Noetherian lattice with " $*$ ". We shall show that "*" must be the trivial multiplication. It will then follow that $L^{\prime}$ with "o" is a Noetherian lattice if and only if $A \leqq M$. Thus Bogart's construction does not hold for arbitrary $A$. Since $L^{\prime}$ is local, the only idempotent elements are $A M$ and $I$. Thus $B^{2}=A M$ for all atoms $B$ in $L^{\prime}$. Since $B * C \leqq B \wedge C=A M$ for all atoms $B, C$ in $L^{\prime}$ with $C \neq B$, it follows that all products and powers of atoms in $L^{\prime}$ are equal to $A M$. Because each element of $L^{\prime}$ different from $I$ or $A M$ is a finite join of atoms of the form $E \vee A M$, where $E$ is principal in $L$ [3, Theorem 1.2], it follows immediately that "*" is the trivial multiplication.

A simple proof of Theorem 5.1 of [1] is as follows. Since each element of $A / A M$ different from $A M$ is a finite join of atoms of the form $E \bigvee A M$, where $E$ is principal in $L$ [3, Theorem 1.2], in particular, $A$ is a finite join of such elements. Since $A / M A$ is modular it follows immediately that $A / A M$ is a complemented modular lattice which satisfies both chain conditions and is thus finite dimensional (see for example [2]).

3. The general case. Let $L$ be a multiplicative lattice and let $M$ be a complete lattice. Elements of $L$ will be denoted by $a, b, c, \cdots$ with the exception that 0 will denote the null element of $L$ and $I$ will denote the unit element of $L$. Elements of $M$ will be denoted by $A$, $B, C, \cdots$ with 0 also denoting the null element of $M$. The usage of 0 will make it clear which null element is intended. In [3], $M$ is defined to be an $L$-module in case there is a multiplication between 
elements of $L$ and $M$, denoted by $a A$ for $a$ in $L$ and $A$ in $M$, which satisfies: (i) (ab) $A=a(b A)$, (ii) $\left(\mathrm{V}_{\alpha} a_{\alpha}\right)\left(\mathrm{V}_{\beta} B_{\beta}\right)=\mathrm{V}_{\alpha, \beta} a_{\alpha} B_{\beta}$, (iii) $I A$ $=A$, and (iv) $0 A=0$, for all $a, a_{\alpha}, b$ in $L$ and for all $A, B_{\beta}$ in $M$.

Let $M$ be an $L$-module. For $B, C$ in $M, B: C$ will denote the largest $d$ in $L$ such that $d C \leqq B$. An element $A$ in $M$ is said to be meet principal in case

$$
(b \wedge(B: A)) A=b A \wedge B
$$

for all $b$ in $L$ and for all $B$ in $M ; A$ is said to be join principal in case

$$
b \vee(B: A)=(B \vee b A): A
$$

for all $b$ in $L$ and for all $B$ in $M$; and, $A$ is said to be principal in case $A$ is both meet and join principal. $M$ is said to be Noetherian if $M$ satisfies the ascending chain condition, is modular, and every element is a join of principal elements. $M$ is said to be an $L$-module with the trivial multiplication if $I B=B$, for all $B$ in $M$, and if $a B=0$, for all $B$ in $M$, where $a \neq I$. For other properties and definitions concerning Noetherian lattice modules, the reader is referred to [4] and [5].

In [5, Lemma 2.1], we gave the lattice-module analog of the characterization of principal elements used in the proof of Theorem 3.1 of [1]. Thus the methods of Theorem 3.1 of [1] may be used to prove the following theorem.

THEOREM 3.3. Let $L$ be a modular multiplicative lattice with maximal (proper) elements, let $M$ be a complete modular lattice with more than one element. Then $M$ can be represented as a Notherian L-module with the trivial multiplication if and only if $L$ has only one maximal element and each element of $M$ is a finite join of atoms.

Proof. Suppose $M$ is a Noetherian $L$-module with the trivial multiplication. As in [1], every nonzero principal element of $M$ is an atom. Since $M$ is Noetherian and every element of $M$ is a join of principal elements, every element of $M$ is a finite join of atoms.

The remainder of the proof consists of substituting $a$ for $A$ and $b$ for $B$ in the first part of the proof of Theorem 3.1 of [1] and substituting $m$ for $M$ and $b$ for $B$ in the second part of the proof of Theorem 3.1 of [1]. Q.E.D.

We can now prove the following theorem.

THEOREM 3.4. Let $L$ be a modular multiplicative lattice with maximal (proper) elements, let $M$ be a Noetherian L-module, let $m$ be a maximal (proper) element of $L$, and let $A$ be an element of $M$. Then

(3.5) $A / m A$ is a Noetherian $L / m-m o d u l e$, and

(3.6) $A / m A$ is a finite-dimensional complemented modular lattice. 
Proof. For $a, b$ in $L / m$, define $a \circ b=a b \vee m$. For $C$ in $A / m A$ and $b$ in $L / m$, define $b * C=b C \vee m A$. It is easily verified that these definitions of multiplication make $L / m$ into a multiplicative lattice and $A / m A$ into a Noetherian $L / m$-module. Thus (3.5) is satisfied. In addition, it is easily seen that " $*$ " is the trivial multiplication between $L / m$ and $A / m A$. Thus, $A / m A$ is a Noetherian $L / m$-module with the trivial multiplication. It then follows from Theorem 3.3 that each element of $M$ is a finite join of atoms. Hence $M$ is a finite-dimensional complemented modular lattice and (3.6) is established. Q.E.D.

\section{REFERENCES}

1. K. P. Bogart, Nonimbeddable Noether lattices, Proc. Amer. Math. Soc. 22 (1969), 129-133. MR 39 \#2667.

2. N. Jacobson, Lectures in abstract algebra. Vol. I. Basic concepts, Van Nostrand, Princeton, N. J., 1951. MR 12, 794.

3. E. W. Johnson, A-transforms and Hilbert functions in local lattices, Trans. Amer. Math. Soc. 137 (1969), 125-139. MR 38 \#5675.

4. J. A. Johnson, A-adic completions of Noetherian lattice modules, Fund. Math. 66 (1970), 347-373.

5. J. A. Johnson and E. W. Johnson, Lattice modules over semi-local Noether lattices, Fund. Math. 68(1970), 187-201.

California Institute of Technology, Pasadena, California 91109

University of Houston, Houston, Texas 77004 